PlastOx 2007 (2009) 237-254

(C) EDP Sciences, 2009

DOI: $10.1051 /$ ptox/2009017

\title{
Influence de l'environnement sur la propagation des fissures de fatigue
}

\author{
G. Hénaff et F. Menan \\ Laboratoire de Mécanique et de Physique des Matériaux, UMR 6617, ENSMA, \\ 1 avenue C. Ader, BP. 40109, 86961 Futuroscope Chasseneuil, France
}

\begin{abstract}
Résumé. Cet article propose tout d'abord une revue des connaissances sur l'influence d'environnements gazeux, et en premier lieu de l'air ambiant, sur la propagation des fissures de fatigue dans les alliages métalliques. Des mises en évidence expérimentales des différentes formes d'interaction entre l'exposition à l'air et la propagation de fissure sont tout d'abord présentées. L'analyse de ces phénomènes est ensuite conduite en considérant deux mécanismes distincts : adsorption des molécules de vapeur puis rupture assistée par l'hydrogène en pointe de fissure. L'applicabilité de ces concepts au cas des milieux aqueux comme la nécessité de prendre en compte le caractère spécifique sont abordés dans une dernière partie.
\end{abstract}

\section{INTRODUCTION}

L'endommagement par fatigue recouvre deux phases à savoir l'amorçage et la propagation de fissure. L'importance relative de ces deux phases est fonction d'un très grand nombre de paramètres liés au matériau ou aux conditions de sollicitation. La phase de propagation peut donc dans certains cas recouvrir une part importante de la durée de vie totale. En outre, l'existence éventuelle de défauts de type fissure ne saurait être écartée dans de nombreuses applications qui doivent dès lors être justifiées en tolérance aux dommages. Pour répondre à cet objectif, il convient entre autres de se doter d'outils permettant de prédire de façon la plus réaliste possible la croissance de tels défauts sous sollicitations de service. Ces sollicitations de service influencent de façon variée la propagation des fissures de fatigue engendrée par le chargement cyclique au même titre que les paramètres métallurgiques. Au cours des cinquante dernières années, de très nombreuses études ont visé à quantifier et tenter de modéliser l'effet des facteurs mécaniques et métallurgiques. Toutefois, très peu d'études se sont penchées sur le rôle joué par l'environnement dans lequel le composant est sollicité, bien qu'il puisse, dans de nombreux cas, engendrer les variations les plus importantes des vitesses de propagation. C'est le cas notamment à l'air ambiant qui constitue l'environnement dans lequel sont sollicitées de nombreuses structures. Il importe donc de baser les méthodes prédictives sur une compréhension des mécanismes physiques régissant les effets couplés de fatigue et d'exposition à l'environnement. Les paragraphes suivants ont donc pour objectif de dresser un état de l'art des connaissances dans ce domaine ainsi que des verrous qui restent à lever pour aboutir à une description pleinement réaliste de ces phénomènes.

\section{PHÉNOMÉNOLOGUE DES INTERACTIONS ENTRE UN ENVIRONNEMENT HUMIDE ET LES AUTRES PROCESSUS AFFECTANT LA RÉSISTANCE À LA FISSURATION}

Le rôle néfaste de l'air ambiant ou de tout autre atmosphère humide, établi depuis longtemps sur la durée de vie totale en fatigue [1,2], n'a été mis en évidence sur la fissuration que relativement récemment (voir par exemple [3, 4]). Les résultats expérimentaux établis dans le domaine des vitesses de propagation moyennes ou élevées montrent que, dès que la pression partielle en vapeur d'eau dépasse une valeur seuil, les vitesses de propagation sont plus élevées que celles mesurées sous environnement inerte [4, 5]. Toutefois le rôle de la vapeur d'eau paraît plus confus dans le domaine des basses vitesses. Ainsi la Figure 1 a présente les résultats obtenus [6] sur un acier structural type E460 pour un rapport de charge $\mathrm{R}=0.1$ et trois environnements, à savoir un vide poussé, considéré comme environnement inerte, l'air 
ambiant et une atmosphère d'azote contenant des traces de vapeur d'eau $(\approx 150 \mathrm{ppm}$.), représentant un environnement intermédiaire entre les deux précédentes atmosphères du point de vue de la teneur en vapeur d'eau. Les vitesses de propagation sous air ne sont en fait que légèrement supérieures à celles obtenues sous vide, avec une accélération d'un facteur 4 environ dans le domaine des vitesses supérieures $\left(\mathrm{da} / \mathrm{dN}>10^{-9} \mathrm{~m} /\right.$ cycle). Près du seuil, la fissure se propage même plus lentement à l'air que sous vide, ce qui va à l'encontre de l'influence supposée néfaste de la vapeur d'eau énoncée précédemment. De la même façon, à peu près dans le même domaine, la propagation est plus rapide sous azote que sous air, et ce bien que la teneur en vapeur d'eau soit nettement plus faible. L'explication réside dans le fait que, dans ce cas précis, la vapeur d'eau n'agit pas uniquement l'endommagement en pointe de fissure mais affecte également des processus comme les effets de fermeture de fissure plus extrinsèques car dépendants du rapport de charge $\mathrm{R}$ et se développant dans le sillage de la fissure [7]. Ces effets de fermeture de fissure peuvent à leur tour être influencés par l'environnement. Afin de rendre compte des interactions complexes entre environnement et fermeture, Suresh et Ritchie $[8,9]$ introduisirent le concept de fermeture induite par l'oxyde. Dans un environnement humide, pour les faibles rapports de charge, le frottement des lèvres de la fissure entre elles engendre la formation d'une surépaisseur d'oxyde du même ordre de grandeur que le déplacement cyclique des lèvres $\triangle \mathrm{CTOD}$, ce qui accentue les effets de fermeture par un effet de «coin» [10]. Selon ces auteurs cependant, l'action de l'environnement au voisinage du seuil se cantonnerait à la seule réduction du facteur d'intensité de contrainte par le biais de l'accroissement du niveau de fermeture. Liaw et al. [11, 12] ont conduit une étude similaire, mais en mesurant l'intensité des effets de fermeture. Ils montrent que la fermeture induite par l'oxyde ne peut pas rendre compte à elle seule des différences de comportement observées entre atmosphères humides et milieu inerte. En effet, après correction des effets de fermeture, les vitesses de propagation mesurées sous argon sec ou hydrogène sec sont respectivement similaires ou plus faibles que celles mesurées sous air. Les auteurs en concluent que, dans de tels environnements gazeux, les cinétiques de propagation sont essentiellement gouvernées par la teneur en vapeur d'eau. Il en résulte que, si le phénomène de fermeture permet de rendre compte de l'influence du rapport de charge sur la propagation au voisinage du seuil, il n'explique pas les différences observées entre un milieu humide et une atmosphère inerte en l'absence d'effet de fermeture. Les tendances dégagées après correction des effets de fermeture dans le cas de l'acier E460 sont d'ailleurs en accord avec ces conclusions, comme le montre la Figure 1 (b). L'air ambiant induit effectivement des vitesses de propagation nettement plus élevées dans toute la gamme explorée. De plus, l'azote n'apparaît plus comme un environnement plus actif que l'air ambiant : en effet le comportement observé sous ce type d'atmosphère est intermédiaire entre l'air et le vide comme attendu. Ce type de démarche, visant à séparer les effets, confirme donc de mettre en évidence un effet néfaste de la vapeur d'eau sur la résistance à la fissuration. Il reste toutefois à éclaircir les mécanismes responsables de cette perte de résistance à la fissuration, ce qui requiert notamment la détermination de la résistance intrinsèque du matériau considéré.

\section{IDENTIFICATION DES MÉCANISMES}

\subsection{Résistance intrinsèque}

La Figure 2(a) présente une compilation de données générées sous vide et corrigées des éventuels effets de fermeture pour différentes nuances d'aciers. Le comportement en fissuration intrinsèque en stade II (considérant la valeur effective du facteur d'intensité de contrainte intégrant les éventuels effets de fermeture) est convenablement décrit par une loi déduite d'approches théoriques basées sur un cumul de dommage en pointe de fissure [13-16] de la forme :

$$
\frac{\mathrm{da}}{\mathrm{dN}}=\frac{\mathrm{A}}{\mathrm{D}_{0}^{*}}\left(\frac{\Delta \mathrm{K}_{\mathrm{eff}}}{\mathrm{E}}\right)^{4}
$$




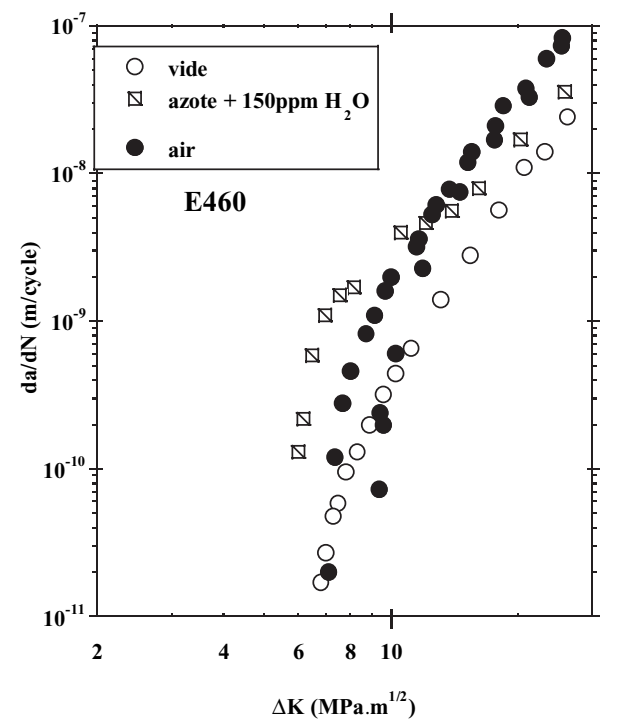

a)

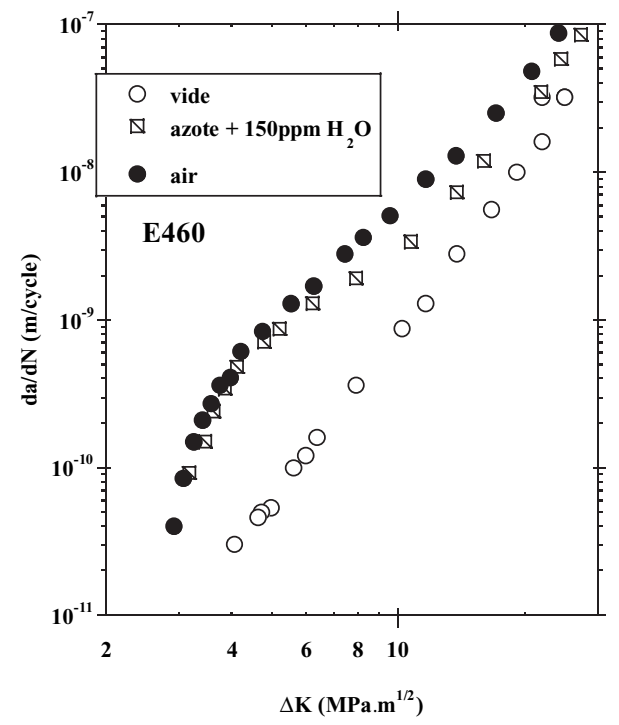

b)

Figure 1. Influence de l'environnement sur le comportement d'un acier E460 : a) sans correction des effets de fermeture, $b$ ) après correction des effets de fermeture.

où $\mathrm{D}_{0}^{*}$ représente la valeur critique du déplacement cumulé en pointe de fissure conduisant à la rupture. La Figure 2(b) présente des données intrinsèques relatives à des aciers et comparées à la courbe moyenne obtenues pour les alliages d'aluminium en fonction de $\frac{\Delta \mathrm{K}_{\text {eff }}}{\mathrm{E}}$. Le paramètre $\mathrm{D}_{0}^{*}$ ne semble dépendre que très légèrement de la nature de la matrice métallique considérée. Les paragraphes suivants examinent les mécanismes proposés pour décrire l'influence de l'environnement et leur prise en compte dans cette loi de propagation.

\subsection{Propagation assistée par l'adsorption}

Les résultats présentés sur la Figure 3 ont été obtenus sur un acier faiblement allié sous très faibles pressions partielles de vapeur d'eau $\left(10^{-3} \mathrm{~Pa}\right)$ et dans des conditions libres d'effets de fermeture [22]. Ils montrent en premier lieu que de très faibles quantités de vapeur sont suffisantes pour induire une accélération de la propagation dès lors que la fréquence est suffisamment basse, d'où la nécessité de garantir des niveaux de vide suffisants pour déterminer la résistance intrinsèque d'un alliage donné. Par ailleurs, la courbe obtenue à $0.2 \mathrm{~Hz}$ montre une dépendance en $\Delta \mathrm{K}_{\text {eff }}^{4}$ comme le comportement intrinsèque obtenu à plus haute fréquence $(35 \mathrm{~Hz})$, avec toutefois des vitesses de propagation 4 fois plus élevées. Pour des fréquences intermédiaires le comportement suit, au voisinage du seuil, la courbe «basse fréquence» puis décroche pour rejoindre progressivement la courbe représentative du comportement intrinsèque.

Ce type de comportement a été attribué à l'adsorption de molécules de vapeur d'eau [22]. De plus ce processus n'affecte pas le mécanisme de base qui peut être décrit par une relation du type :

$$
\frac{\mathrm{da}}{\mathrm{dN}}=\frac{\mathrm{A}}{\mathrm{D}_{1}^{*}}\left(\frac{\Delta \mathrm{K}_{\mathrm{eff}}}{\mathrm{E}}\right)^{4}
$$

L'hypothèse est que l'adsorption des molécules de vapeur d'eau diminue la valeur du paramètre $\mathrm{D}^{*}$ en fonction du taux de couverture $\theta$ défini comme suit: $\theta=0$ en l'absence d'adsorption, $\theta=1$ lorsque l'adsorption est saturante, c'est-à-dire lorsque tous les sites disponibles sur la surface sont occupés par 


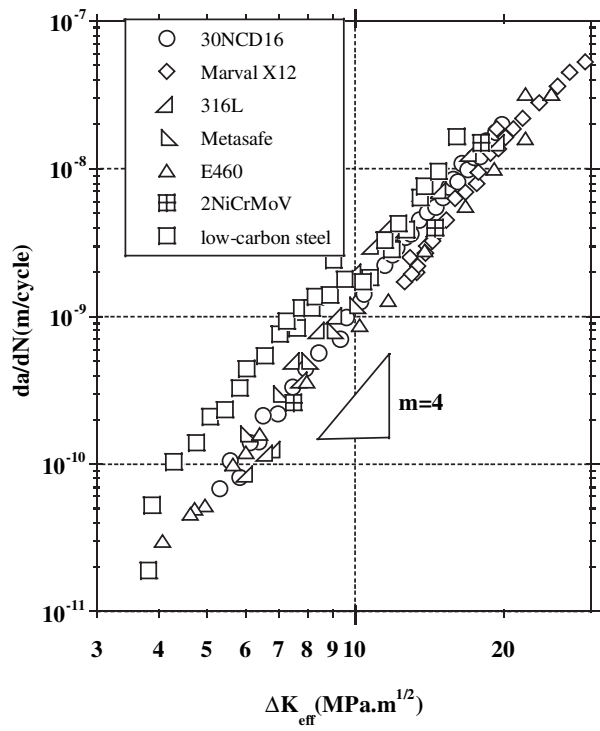

a)

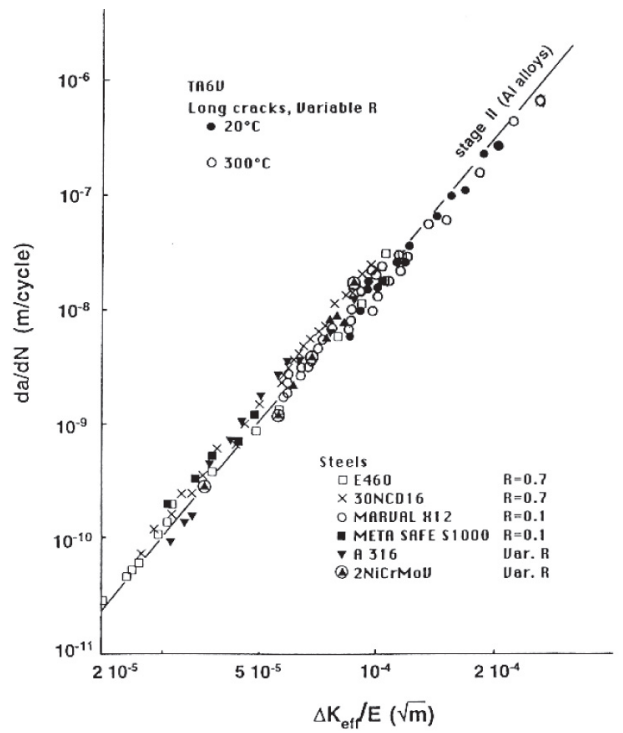

b)

Figure 2. a) Comportement intrinsèque de différents nuances d'aciers $[6,17-20]$, b) comparaison avec d'autres familles d'alliages [21].

une molécule adsorbée. L'hypothèse selon laquelle l'adsorption serait saturante à température ambiante est raisonnable compte tenu de la faible désorption. Les résultats de la Figure 3 peuvent donc être interprétés de la manière suivante. A haute fréquence, l'incrément de propagation se produit avant que les molécules n'aient le temps d'atteindre la pointe de fissure et d'adsorber sur la surface $(\theta=0$ et $\left.\mathrm{D}^{*}=\mathrm{D}_{0}^{*}\right)$. Lorsque l'on abaisse la fréquence, de plus en plus de temps est disponible pour former une monocouche adsorbée en pointe de fissure. Le taux de couverture augmente jusqu'à atteindre $\theta=1$ et $\mathrm{D}^{*}=\mathrm{D}_{1}^{*}$ à saturation. Dans le régime intermédiaire on suppose que $\mathrm{D}^{*}$ est relié à $\theta$ par la relation :

$$
\mathrm{D}^{*}=\frac{\mathrm{D}_{0}^{*} \mathrm{D}_{1}^{*}}{\mathrm{D}_{1}^{*}+\theta\left(\mathrm{D} 0_{1}^{*}-\mathrm{D}_{1}^{*}\right)}
$$

Un modèle a également été développé pour décrire l'évolution de $\theta$ en fonction du temps (ou de la fréquence) [22]. La pression dans un volume de contrôle en pointe de fissure, qui gouverne les cinétiques d'adsorption, résulte d'une compétition entre, d'une part le transport des espèces actives en pointe de fissure, d'autre part la consommation de ces molécules par le processus d'adsorption. La phase de transport est modélisée en prenant en compte l'effet d'impédance de la fissure selon la proposition de Bradshaw [4]. La fissure est assimilée à un étroit canal reliant le volume extérieur, où règne une pression donnée, à un volume de contrôle situé en pointe de fissure où la pression est plus faible. Cette différence de pression, analogue à une différence de potentiel électrique, est estimée selon l'hypothèse d'un écoulement de type moléculaire le long de la fissure [22]. Le taux de molécules consommées par adsorption est, quant à lui, estimé à partir de l'hypothèse selon laquelle dès qu'une molécule vient frapper la surface, elle s'y adsorbe instantanément. Les résultats obtenus, représentés sur la Figure 3 par un trait continu, montrent, là encore, un excellent accord avec les données expérimentales et confirment le rôle prépondérant joué par la vapeur d'eau. Ce modèle a également été utilisé pour décrire la propagation sous hydrogène sec dans des aciers faiblement alliés [12, 23] et sous faibles pressions partielles de vapeur d'eau dans le cas d'un alliage de titane Ti-6Al-V à $300^{\circ} \mathrm{C}$ [23, 24]. Une approche similaire a récemment été reprise par Ro et al. [25]. 


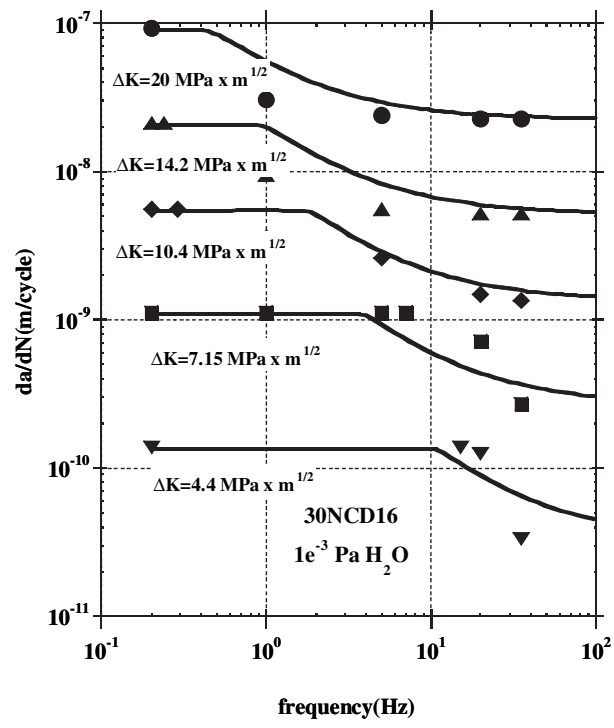

a)

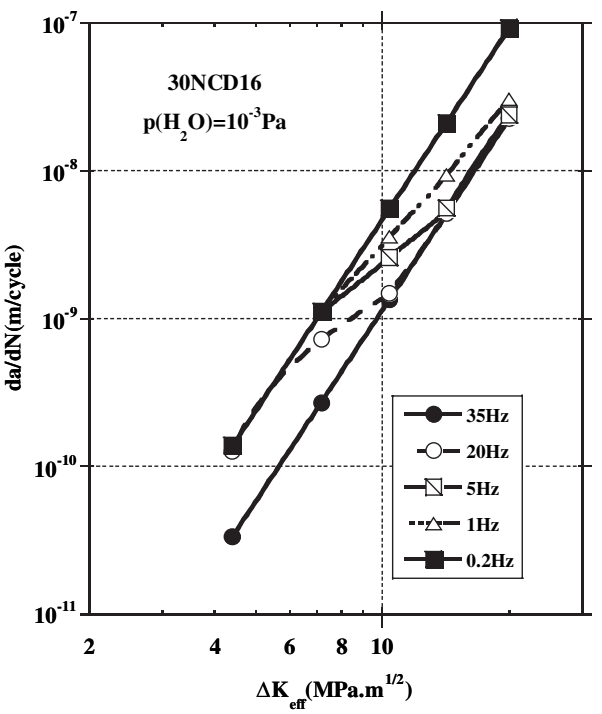

b)

Figure 3. Propagation sous très faible pression en vapeur d'eau dans un acier faiblement allié : a) influence de la fréquence ; b) da/dN vs. $\Delta \mathrm{K}_{\mathrm{eff}}[22]$.

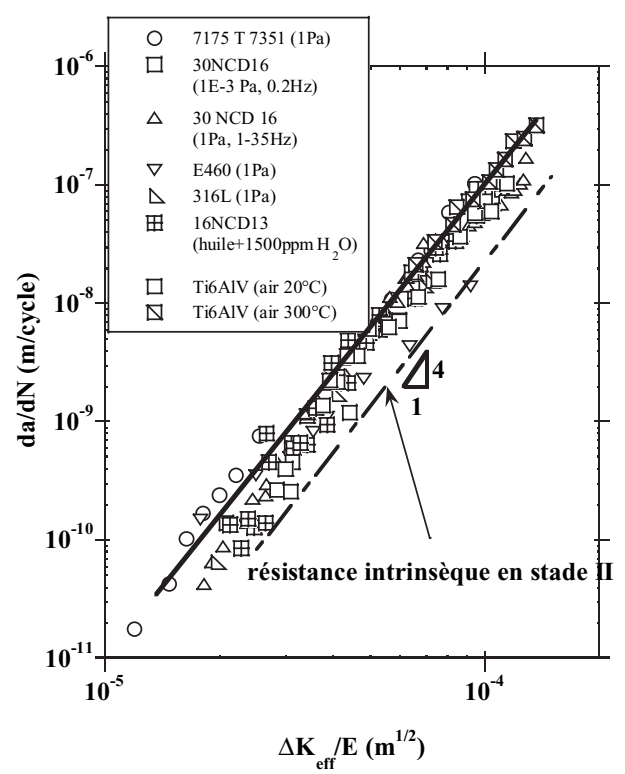

Figure 4. Compilation de données sur la propagation contrôlée par l'adsorption de vapeur d'eau [22].

Une compilation de résultats obtenus sur des alliages de nature différente et sous des conditions d'environnement variées et présentée en fonction du paramètre $\Delta \mathrm{K}_{\mathrm{eff}} / \mathrm{E}$ sur la Figure 4 [22] montre que le concept de propagation assistée par l'adsorption constitue un phénomène commun à de nombreux alliages métalliques suivant un processus compatible avec le modèle AIDE (Adsorption-Asssited Decohesion) proposé par Lynch [26]. 


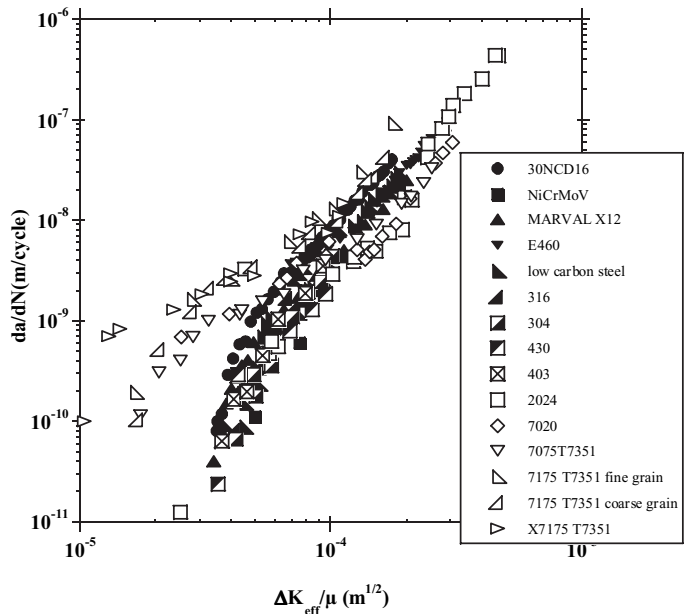

a)

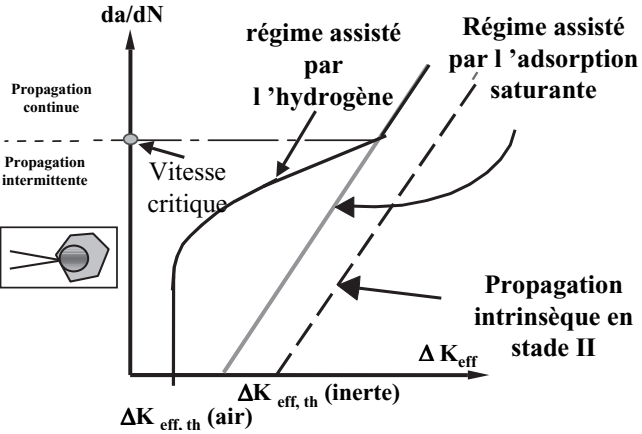

b)

Figure 5. a) Compilation de données sur le comportement au voisinage du seuil à l'air ambiant dans les aciers et les alliages d'aluminium ; b) Mécanisme d'action de l'air ambiant sur la propagation des fissures de fatigue.

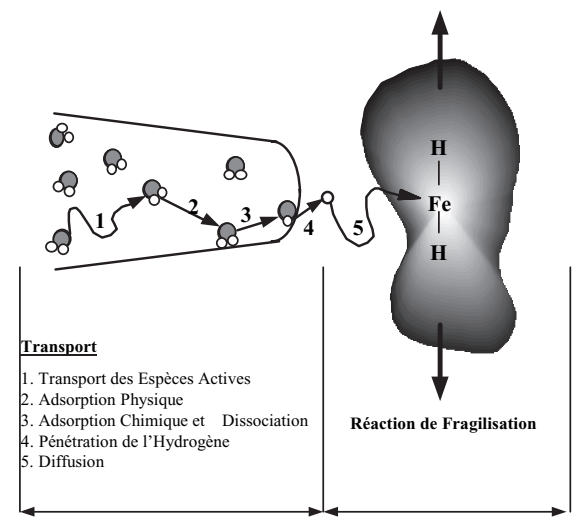

Figure 6. Mécanisme de propagation assistée par l'hydrogène selon Wei [27].

\subsection{Propagation assistée par l'hydrogène}

La Figure 5 présente une compilation de données effectives de propagation obtenues sur deux classes d'alliages (aciers et alliages d'aluminium) à l'air ambiant exprimée en fonction de $\Delta \mathrm{K}_{\mathrm{eff}} / \mathrm{E}$. Si pour les aciers les données tombent dans une bande de dispersion relativement étroite, les alliages d'aluminium présentent quant à eux une plus grande disparité, notamment au voisinage du seuil. Ces courbes présentent néanmoins des caractéristiques communes, à savoir une tendance à retrouver un régime de propagation caractérisé par une pente 4 pour les vitesses supérieures, un changement de pente caractéristique $(\mathrm{m}=4 \rightarrow \mathrm{m} \approx 2.7)$ en dessous d'une vitesse de fissuration critique $\left(10^{-9} \mathrm{~m} /\right.$ cycle $\leq$ $\left(\frac{\mathrm{da}}{\mathrm{dN}}\right)_{\mathrm{cr}} \leq 5 \times 10^{-8} \mathrm{~m} /$ cycle) et une diminution brutale des vitesses de propagation lorsque $\Delta \mathrm{K}_{\mathrm{eff}} \rightarrow$

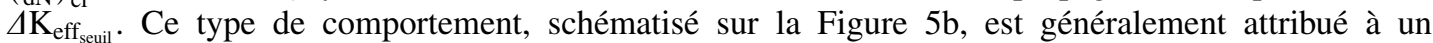
phénomène de fragilisation par l'hydrogène en pointe de fissure selon un processus séquentiel illustré sur la Figure 6 [27]. 
Selon Achter [28] et Bowles [29], l'entrée en action d'un processus de fragilisation requiert un temps d'exposition suffisamment long. L'étape d'adsorption serait alors le seul facteur limitant pour le déclenchement du mécanisme lié à l'hydrogène. Or les résultats expérimentaux montrent que l'obtention d'un état d'adsorption saturante ne constitue nullement une condition suffisante. Part conséquent d'autres étapes limitantes doivent être considérées, en particulier la cinétique de dissociation des molécules adsorbées constitue un autre facteur critique. Selon Wei [27] la constante de réaction de surface des alliages d'aluminium avec la vapeur d'eau serait supérieure de plusieurs ordres de grandeurs $\left(10^{8}\right.$ à $\left.10^{9}\right)$ à la constante de décomposition sur acier, conduisant à deux situations caractéristiques, à savoir un processus contrôlé par le transport dans un cas et un processus contrôlé par la réaction de surface dans l'autre cas.

L'hydrogène peut diffuser extrêmement rapidement au sein du réseau. Cependant, le transport de l'hydrogène par les dislocations mobiles sous formes d'atmosphères de Cottrell se révèle beaucoup plus rapide que la diffusion de réseau [30-33]. Considérant le comportement au niveau du seuil comme une compétition entre l'avance de la fissure et le drainage de l'hydrogène par les dislocations en pointe de fissure, Marchand et al. [34] déduisent deux vitesses critiques vis à vis de ce processus :

- la limite supérieure correspond au cas où la fissure se propage si rapidement qu'il devient impossible d'atteindre une concentration critique en hydrogène en pointe de fissure pour induire un dommage significatif ;

- à la limite inférieure les dislocations mobiles ne sont plus suffisamment nombreuses pour drainer une quantité critique d'hydrogène produit sur les surfaces de rupture avant l'incrément suivant de propagation engendré par la déformation cyclique en pointe de fissure.

Le processus de propagation assistée par l'hydrogène constituerait donc un phénomène transitoire, et le seuil apparent observé à l'air serait avant tout représentatif de la disparition du processus lié à l'hydrogène. Si le rôle de l'hydrogène sur les propriétés mécaniques des alliages métalliques, et principalement des alliages ferreux, a été le sujet d'une volumineuse littérature [57-60], peu d'études ont spécifiquement porté sur le rôle de l'hydrogène dans la propagation des fissures de fatigue. La fragilisation par l'hydrogène suppose, comme mentionné plus haut, d'atteindre localement une concentration critique en hydrogène. Cette condition peut être plus facilement atteinte au voisinage du seuil par la localisation de la déformation et par le passage d'une progression cycle par cycle de la fissure à un régime de propagation intermittente.

Les théories sur la rupture assistée par l'hydrogène sont généralement regroupées en deux grandes familles :

- les théories HEDE (Hydrogen-Enhanced DEcohesion);

- les approches du type HELP (Hydrogen-Enhanced Localised Plasticity).

Les théories HEDE reposent sur l'hypothèse que l'hydrogène favorise l'apparition de microfissure par un abaissement de la contrainte de cohésion du réseau [35-38]. Le caractère ductile des processus de fissuration liés à l'hydrogène, mis en évidence notamment par Beachem [39] constitue le support des théories du type HELP. Différents auteurs ont depuis mis en évidence, par des observations de déformation in-situ notamment, que, pour des métaux de structure cubique centrée, cubique face centrée ou hexagonale compacte, l'hydrogène favorise la création et le mouvement des dislocations et par ce biais augmente localement de façon importante le taux de déformation au voisinage de la surface de rupture [40-42]. Ces observations ont également mis en évidence la corrélation entre l'activité des dislocations en pointe de fissure et la présence d'hydrogène dans l'atmosphère [43, 44]. Un autre facteur est lié la localisation de la déformation suivant un nombre limité de systèmes de glissement. Ce phénomène permet de concilier la perte de ductilité macroscopique avec des micromécanismes de déformation et de rupture intrinsèquement ductiles [45]. Brass et Chêne notent toutefois que dans les alliages ferreux et les alliages de nickel, l'hydrogène favorise à la fois la localisation et la fragilisation [46]. La localisation de la déformation induite par l'hydrogène pourrait également s'expliquer par le fait que l'hydrogène affecte la contrainte requise pour activer une source au voisinage immédiat de la pointe de fissure et, une fois la source activée, il serait plus favorable de la maintenir 
active que d'activer du glissement sur d'autres systèmes qui contribueraient à l'émoussement comme sous environnement inerte [41]. Un autre argument attribue à l'hydrogène la capacité à favoriser le caractère «coin» des dislocations et par ce biais à limiter le glissement dévié et donc favoriser la localisation [41, 42]. Dans le cas où la déformation est intrinsèquement localisée, Hanninen et al. [47] observent malgré tout un effet de l'hydrogène sur l'activité des dislocations au sein de ces bandes de localisation. Il convient enfin de noter que la localisation de la déformation est également incluse dans la description apportée par Lynch de l'effet de l'adsorption [48]. En effet, bien que l'adsorption soit un phénomène de surface, elle peut affecter les processus de déformation en pointe de fissure et favoriser la progression de la fissure par un processus de glissement alterné et coalescence de micro-cavités. De ce fait l'émoussement et les déformations en pointe s'en trouvent réduits par rapport à un environnement inerte.

Ces observations ont pu être validées et intégrées dans des modèles pour décrire la corrosion sous contrainte comme celui de Magnin par exemple [49, 50]. Cependant peu de travaux ont porté spécifiquement sur la pertinence de ces mécanismes en fatigue, notamment en fissuration où la zone affectée par ces processus est extrêmement petite. Maier et Kæsche [51] ont montré, dans le cas d'un acier faiblement allié sollicité en fatigue, que le développement de cellules de dislocations caractéristiques n'était pas lié à la présence d'hydrogène mais était favorisé par l'hydrogène facilitant le mouvement des dislocations. Magnin et al. [52] notent que l'hydrogène diminue effectivement la propension au glissement dévié de monocristaux de nickel orientés pour le glissement simple et sollicités en déformation cyclique. Le régime de saturation est ainsi atteint plus rapidement en présence d'hydrogène. Sur la base d'observations in-situ de la pointe de la fissure, McEvily et Gonzalez Vazquez [53] ont proposé de rendre compte de l'influence de l'environnement sur la propagation en fatigue par un émoussement moins prononcé à l'air que sous vide. L'hydrogène induisant une localisation de la déformation en pointe, une énergie moins importante est dissipée en émoussement et par conséquent une part plus grande de l'énergie transmise participe directement à la propagation, d'où des vitesses plus élevées. Davidson et Lankford [54] étaient parvenus à des conclusions similaires. Ils ajoutaient cependant que la contribution du mode II pouvait être diminuée dans l'air ambiant, ce qui, là encore, induirait des vitesses plus rapides puisque toute l'énergie de déformation est activée en mode I. Enfin, MacEvily et al. [55] observent, dans le cas d'un acier inoxydable austénitique 304, que la densité de dislocations au sein de la zone plastifiée en tête de fissure est beaucoup plus élevée et plus homogène sous vide que sous air. Cependant ce type d'observations ne permet pas de distinguer la part de l'hydrogène du rôle de l'adsorption suivant le mécanisme proposé par Lynch qui prédit également moins d'émoussement.

Il faudrait enfin se garder d'opposer les théories de type HEDE et HELP. En effet si les dernières permettent de rendre compte des modifications de plasticité en pointe de fissure décrite ci-dessus, les mécanismes de type HEDE peuvent expliquer les vitesses de propagation plus élevées sous environnement hydrogénant par l'introduction d'un endommagement spécifique.

A défaut de disposer d'un modèle décrivant les processus physiques impliqués, on doit se satisfaire de lois de propagation plus ou moins empiriques. On peut ainsi décrire convenablement la propagation effective au voisinage du seuil à l'aide de la relation en sommant simplement les contributions respectives de l'adsorption et du mécanisme assisté par l'hydrogène:

$$
\frac{\mathrm{da}}{\mathrm{dN}}=\frac{\mathrm{A}}{\mathrm{D}_{1}^{*}}\left(\frac{\Delta \mathrm{K}_{\mathrm{eff}}}{\mathrm{E}}\right)^{4}+\frac{\mathrm{B}}{\sigma \mathrm{E}}\left(\Delta \mathrm{K}_{\mathrm{eff}}^{2}-\Delta \mathrm{K}_{\text {eff,seuil }}^{2}\right)
$$

Des exemples d'application sont présentés sur la Figure 7.

\subsection{Contribution des deux mécanismes élémentaires dans les alliages intermétalliques}

Les deux mécanismes décrits précédemment peuvent avoir des effets quantitativement différents sur les vitesses de propagation en fonction de l'alliage considéré. C'est notamment le cas des alliages 

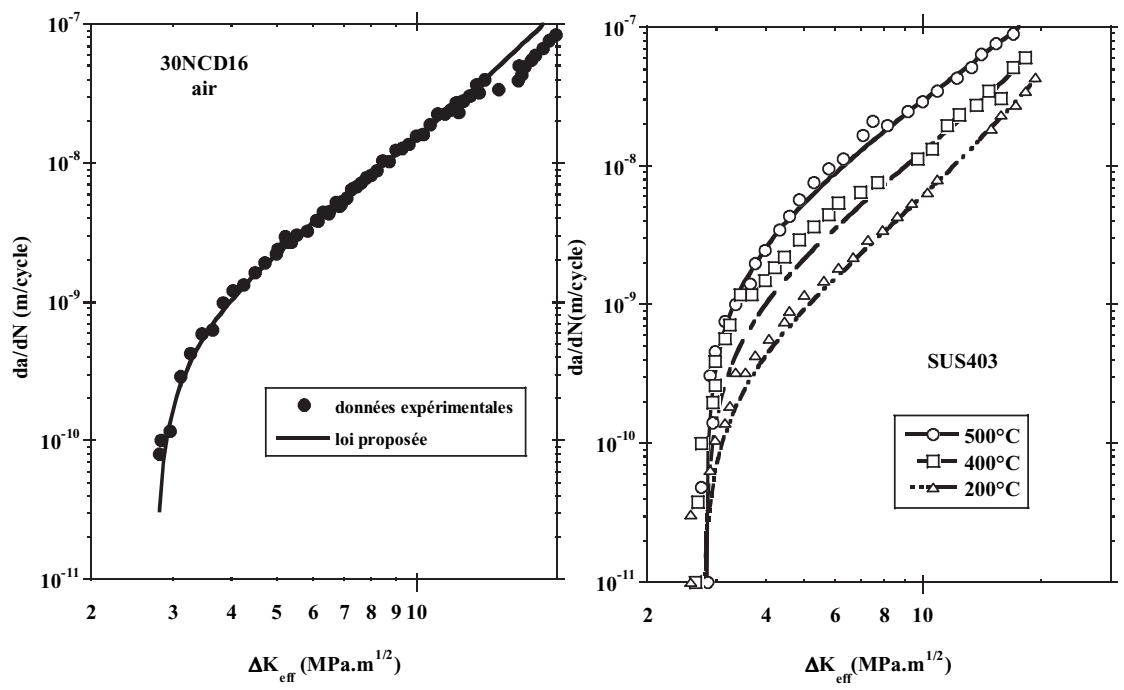

Figure 7. Loi de propagation à l'air ambiant appliqué au cas de l'acier 30NCD16 [20] (a) et à un acier inoxydable martensitique à différentes températures [56] (b).

basés sur les composés intermétalliques FeAl et TiAl. Ces matériaux sont connus pour exhiber une sensibilité à l'environnement sous chargement monotone [57, 58] qui se manifeste par une perte de ductilité attribuée à un effet fragilisation par l'hydrogène. La Figure 8 présente les courbes de fissuration obtenues pour l'alliage $\mathrm{FeAl} 40$ grade 3 à l'air et sous vide dans la gamme $25^{\circ} \mathrm{C}-400^{\circ} \mathrm{C}$ [59] (Figure 8a), et des atmosphères intermédiaires du point de vue de la teneur en vapeur d'eau (Figure 8b) [60]. La perte de résistance à la fissuration à l'air paraît globalement indépendante de la température et se traduit par un décalage des courbes vers des vitesses plus élevées pour les faibles valeurs de $\Delta \mathrm{K}$. Par ailleurs, les atmosphères à teneur en vapeur d'eau intermédiaire, conduisent à des vitesses de propagation comparables à celles obtenues l'air, sauf pour l'atmosphère d'oxygène qui elle conduit à un comportement similaire au vide. Ce dernier effet est attribué à une adsorption compétitive des molécules d'oxygène $[57,61]$ qui limite la possibilité l'adsorption de vapeur d'eau. L'oxygène gazeux inhibe donc dans ce cas l'action de la vapeur d'eau sans pour autant induire d'effet par lui-même. Les alliages TiAl présentent un comportement très différent sous les mêmes environnements. En effet on note un changement marqué dans l'allure de la courbe de fissuration se traduisant par des vitesses nettement plus élevées à l'air par rapport au vide quelle que soit la température. En outre, sous atmosphères intermédiaires, on observe un comportement intermédiaire entre l'air et le vide. Ceci montre que la vapeur d'eau reste l'élément actif, y compris à haute température, et que de plus l'oxygène n'inhibe pas l'action de la vapeur d'eau, même à haute température. L'analyse détaillée de ces comportements conduit à attribuer l'action de l'air dans $\mathrm{FeAl}$ à un rôle prépondérant de l'adsorption [62, 63], alors que dans TiAl c'est un mode de fissuration assistée par l'hydrogène qui prévaudrait [64].

\section{PROPAGATION DE FISSURE EN SOLUTION}

\subsection{Quelques spécificités de la fatigue en milieu aqueux}

Il est d'usage d'analyser la propagation de fissure de fatigue en milieu aqueux a priori corrosif selon trois comportements caractéristiques schématisés sur la Figure 10. Le comportement de la Figure 10a, dit de «fatigue-corrosion pure», résulte d'une synergie entre l'endommagement généré par la déformation 


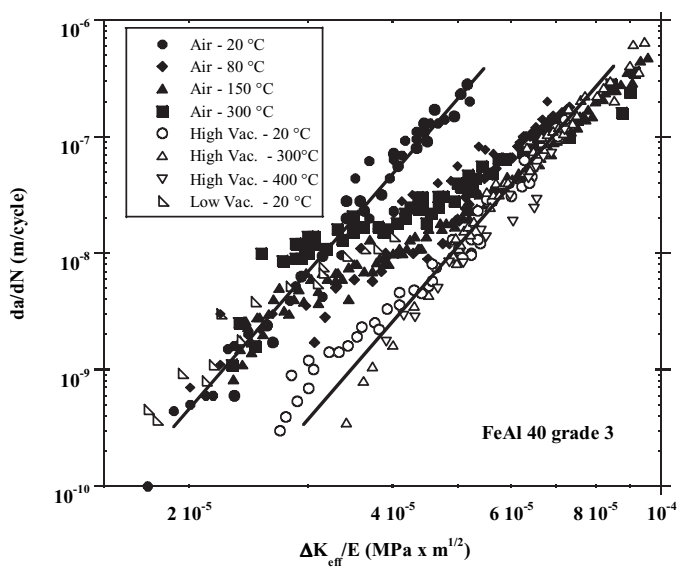

a)

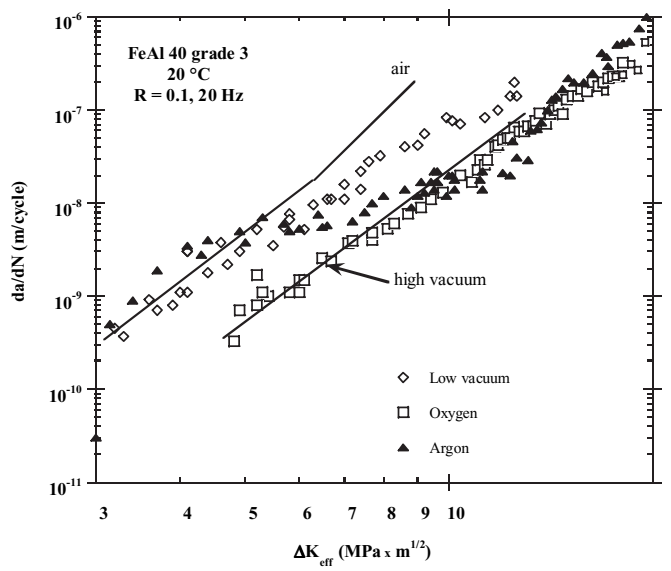

b)

Figure 8. Influence of environment on fatigue crack growth in the $\mathrm{FeAl}$ grade 3 alloy at room-temperature (a) in air and vacuum, and (b) in intermediate atmospheres $[60,63,65]$.

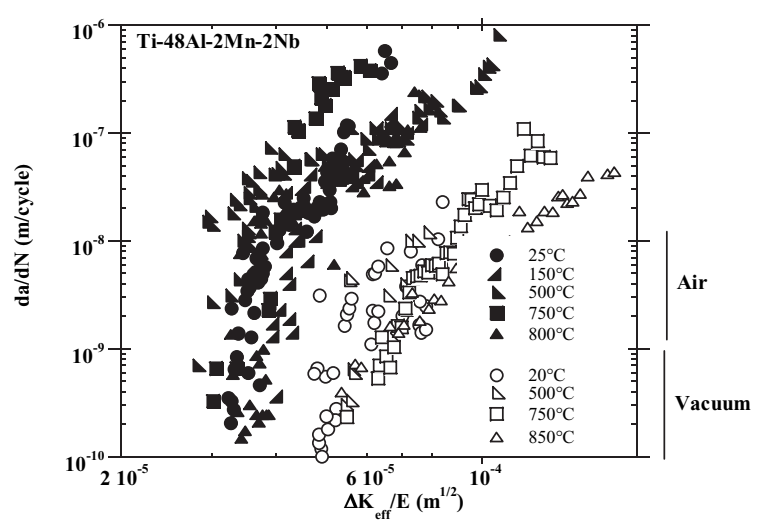

(a)

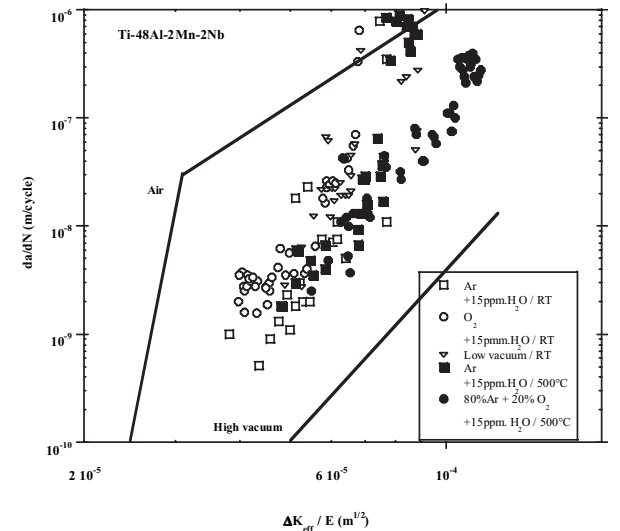

(b)

Figure 9. Influence of environment on fatigue crack growth in the Ti-48Al-2Mn-2Nb alloy (a) in air and vacuum, and (b) in intermediate atmospheres [60, 64].

cyclique en pointe de fissure et l'exposition au milieu corrosif, à la différence du comportement b) qui lui résulte d'un superposition d'un comportement de fatigue «pure » avec un mode de corrosion sous contrainte lorsque le facteur d'intensité de contrainte maximale $\mathrm{K}_{\max }$ excède la valeur seuil $\mathrm{K}_{\mathrm{ISCC}}$. (le comportement c) constitue une combinaison des deux premiers). On notera que le comportement à l'air ambiant au voisinage du seuil relève de la catégorie a) et ne saurait donc constituer un environnement de référence pour évaluer la nocivité d'un milieu aqueux du point de la fissuration. C'est pourquoi, comme le note Huneau et Mendez [66], la confrontation directe des vitesses mesurées dans un milieu corrosif et l'air ambiant peut conduire à des interprétations erronées. Ainsi, dans le cas d'un acier HLE en solution saline et sous protection cathodique, on s'attend à ce que l'hydrogène généré au voisinage de la surface soit la source d'un effet fatigue-corrosion important. Or l'examen de la Figure 11 montre que la différence de vitesse de propagation entre les deux milieux est peu marquée à faible $\Delta \mathrm{K}$ et augmente avec $\Delta \mathrm{K}$, ce qui laisse supposer un effet «hydrogène » peu prononcé à faible $\Delta \mathrm{K}$. Cependant la confrontation avec les données sous vide montre qu'en fait, à l'air comme en solution saline, l'effet d'environnement est plus important à faible $\Delta \mathrm{K}$ et diminue progressivement lorsque $\Delta \mathrm{K}$ augmente. 


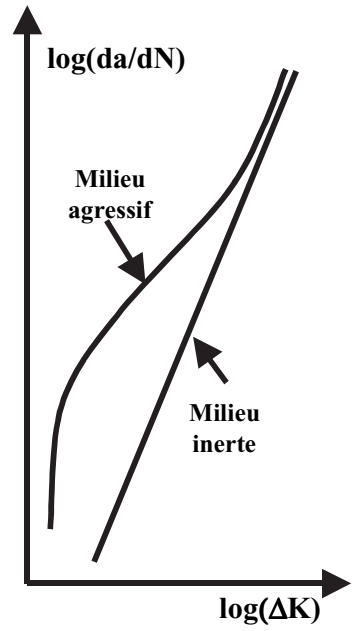

a)

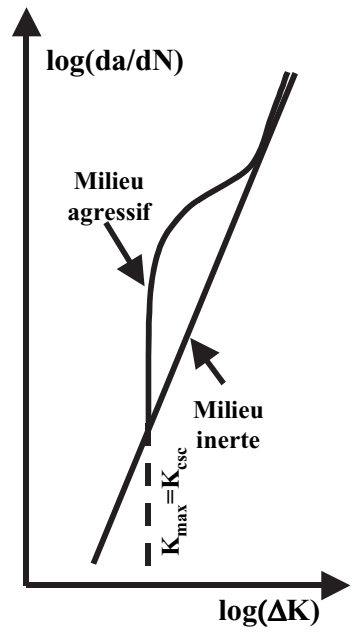

b)

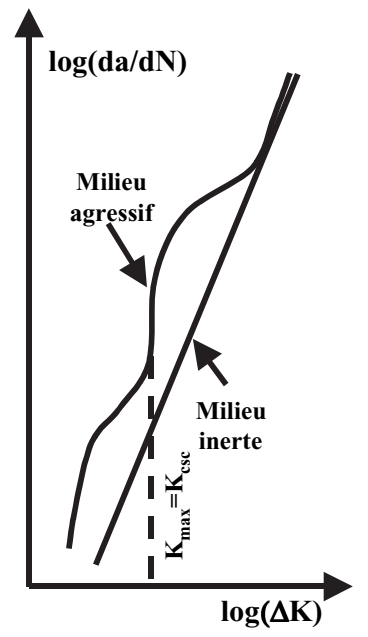

c)

Figure 10. Différents types de comportement en fissuration par fatigue-corrosion : a) fatigue-corrosion pure, b) superposition fatigue/corrosion sous contrainte, c) comportement mixte.

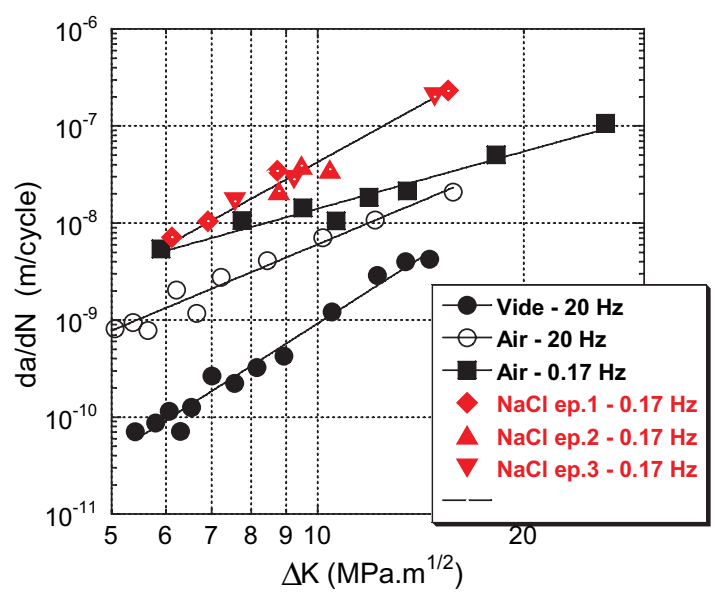

Figure 11. Influence de la fréquence sur les vitesses de propagation dans un acier HLE à l'air et en solution saline sous protection cathodique [66].

Il apparaît au travers de cet exemple que les concepts liés à un phénomène de fragilisation par l'hydrogène vont s'appliquer à la propagation en milieu corrosif. Toutefois, de nouveaux processus susceptibles d'affecter les cinétiques des différentes étapes mises en jeu, comme la repassivation des surfaces de la pointe de fissure, soit la formation d'une couche d'oxyde empêchant l'accès de l'hydrogène à la surface fraîche du métal $[68,69]$, sont également à considérer. En outre ces processus dépendent a priori fortement du couple métal/milieu considéré et des cinétiques de repassivation. C'est le principe du modèle de dissolution/repassivation proposé par Ford [70]. Selon le modèle, initialement développé pour la corrosion sous contrainte et étendu aux chargements cycliques, le film protecteur est rompu lors de la mise en charge. La dissolution des surfaces ainsi fraîchement créées contribue directement à l'avancée de la fissure jusqu'à ce qu'un nouveau film passivant se forme sur ces surfaces. La vitesse de propagation en fonction du temps est alors reliée à la vitesse de déformation en pointe de 


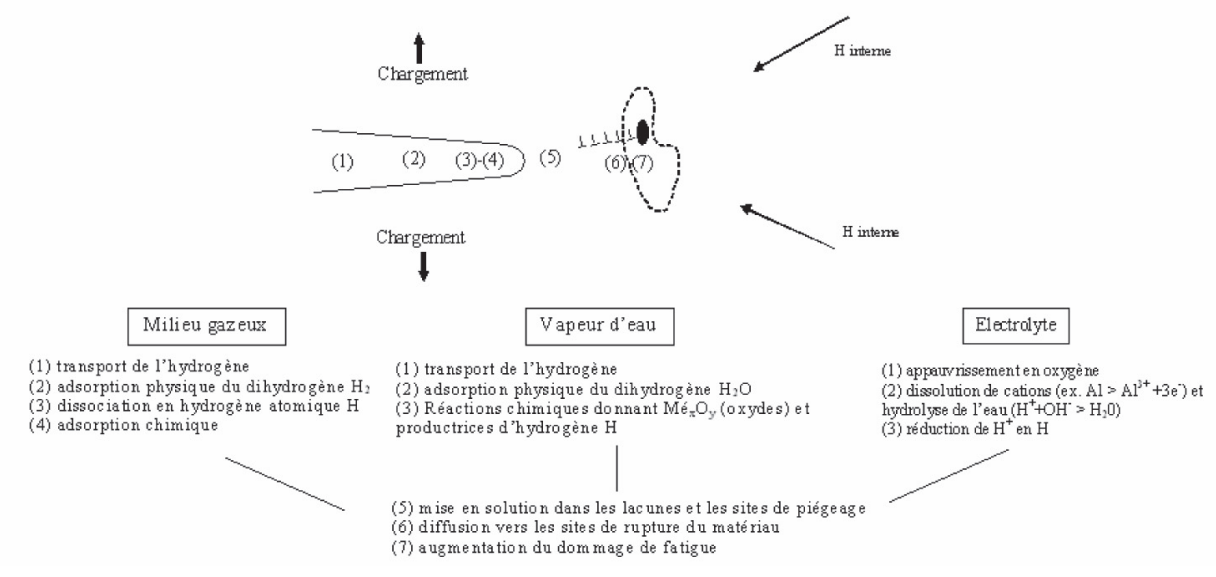

Figure 12. Étapes d'apport d'hydrogène au sein de la zone plastique en pointe de fissure pour l'hydrogène gazeux, la vapeur d'eau et les milieux aqueux [67].

fissure $\dot{\varepsilon}$ :

$$
\frac{\mathrm{da}}{\mathrm{dt}}=\mathrm{A} \times \dot{\varepsilon}_{\mathrm{ct}}^{\mathrm{n}}
$$

A et $\mathrm{n}$ sont des constantes dépendantes du matériau, des conditions environnementales en pointe et de la cinétique de repassivation. On notera qu'un tel modèle ne considère pas d'influence de la dissolution sur la plasticité cyclique. Par ailleurs, les similitudes observées dans de nombreux cas, tant en terme d'accélération de la propagation que de mécanismes de rupture, entre les solutions aqueuses et les atmosphères gazeuses, pour lesquelles il n'a pas d'électrolyte présent en pointe, suggèrent que ce sont les processus liés à l'hydrogène généré par les réactions électrochimiques qui sont prédominants suivant des mécanismes (HELP ou HEDE) qui restent là encore à préciser. Ainsi Ro et al. [71, 72] montrent, dans le cas d'alliages $\mathrm{Al}-\mathrm{Cu}-\mathrm{Li}-\mathrm{Mg} / \mathrm{Li}$ ayant une propension à développer des modes de propagation fortement cristallographiques sous environnement inerte conduisant à la formation de facettes proches de $\{111\}$, que l'air ambiant comme une solution $3.5 \% \mathrm{NaCl}$ ne conduisent plus à la formation de ce type de facettes mais engendre un changement marqué des surfaces de rupture, ce qu'un modèle de type dissolution/repassivation ne saurait expliquer seul. Dans ces environnements, les surfaces sont constituées de deux faciès caractéristiques, à savoir des facettes présentant des marches type de clivage et de larges plages planes suivant des plans de hauts indices. Or, selon ces auteurs, un mécanisme de type HELP aurait avant tout pour effet d'accentuer la fissuration suivant des bandes de glissement observée sous environnement inerte [72]. L'hypothèse retenue serait plutôt que d'une part l'hydrogène favoriserait la formation de facettes avec présence de marches accompagnée de plasticité en pointe et que les plages planes résulteraient de la combinaison de fortes contraintes de traction en pointe et du piégeage de l'hydrogène au sein des structures de dislocations produites par la déformation cyclique.

En milieu aqueux, différents paramètres sont en outre susceptibles d'influencer les vitesses de propagation, notamment la nature et concentration des éléments en solution (oxygène dissous, ions, inhibiteurs de corrosion...), le potentiel électrochimique, le $\mathrm{pH}$, la viscosité de la solution et sa vitesse de circulation [73]. Les milieux aqueux constituent un chemin électrolytique favorable aux échanges d'électrons, donnant naissance à des réactions électrochimiques entre le métal et la solution (dissolution anodique, formations de films d'oxydes, production d'hydrogène... etc.) [74]. La pointe de fissure constitue en outre un milieu confiné. L'accès de la solution peut donc être difficile, ce qui réduit le renouvellement des espèces chimiques. Par conséquent la solution (composition, potentiel électrochimique, $\mathrm{pH}$ ) en pointe peut être très différente de la chimie de la solution environnante $[69,75]$. 
La chimie et composition de la solution au voisinage d'une fissure constituent donc un nouveau domaine d'étude à part entière $[74,76,77]$. Deux points essentiels ressortent: le milieu est généralement plus acide en pointe de fissure car les conditions électrochimiques sont très productrices d'hydrogène, et la solution peut se désaérer du fait d'un apport d'oxygène limité.

La fissuration en solution aqueuse peut donner naissance à de nouvelles sources de fermeture de fissure, principalement par effet «coin», dues à la compression du liquide environnant [78, 79], aux produits de corrosion $[75,78,80,81]$ mais aussi aux grains déchaussés ou détachés de la pointe de fissure par corrosion intergranulaire [78]. On définit ici les produits de corrosion comme les espèces résultant des réactions entre le métal et le milieu et présentes en solution, contrairement à un oxyde qui se crée à la surface du métal. La fermeture due à la compression du liquide a surtout été traitée pour la fissuration dans des milieux non aqueux très épais comme les huiles de lubrification [79]. Par ailleurs les changements de conditions de chargement et/ou de milieu en cours de propagation peuvent donner lieu à des effets transitoires importants sur les vitesses de fissuration. Ainsi Miller, Hudak et Wei [82] ont montré sur un acier 4340 dans l'eau distillée lors d'un essai de fissuration interrompu pendant une nuit un effet « retard» sur la courbe donnant la longueur de fissure en fonction du nombre de cycles pendant environ 3000 cycles. De grandes précautions doivent donc être prises lors de la conduite d'essais en milieu aqueux [83, 84].

Enfin, un type particulier de fissures courtes est à considérer en environnement aqueux, à savoir les fissures chimiquement courtes [85]. Ces fissures chimiquement courtes se caractérisent par des conditions chimiques et électrochimiques en pointe différentes des conditions rencontrées pour les fissures dites «longues». Pour une microstructure et un environnement donnés les vitesses de fissuration ne dépendent alors plus uniquement du chargement mécanique mais aussi de la longueur de fissure. Pour l'alliage 2024-T3, Piascik et Willard [86] n'observent aucun effet de fissure chimiquement courte en solution aqueuse à $1 \% \mathrm{NaCl}$ alors que Wan et al. [87] observent une augmentation des vitesses d'un facteur 2 par rapport aux fissures longues en solution aqueuse à 3\% $\mathrm{NaCl}$. Wan et al. [86] ont surtout démontré que ces effets de fissures courtes n'apparaissent que pour certaines conditions précises de chargement et d'environnement (amplitude $\Delta \mathrm{K}$, rapport $\mathrm{R}$, concentration en oxygène dissous. .. etc.).

\subsection{Interaction chargement - microstructure - environnement : facteurs d'influence}

\subsubsection{Influence de la microstructure}

Pour un chargement et un environnement donnés, deux microstructures distinctes d'un même alliage peuvent présenter des sensibilités différentes à la fatigue-corrosion. A titre d'exemple Gingell et King [88] ont analysé l'influence de la fréquence sur la fissuration de l'alliage d'aluminium 7150$\mathrm{T} 651$ dans le sens $\mathrm{S}-\mathrm{L}$, à $\mathrm{R}=0.3$, en solution saline à $2.5 \% \mathrm{NaCl}+0.5 \% \mathrm{Na}_{2} \mathrm{CrO}_{4}(\mathrm{pH}=3)$ pour deux microstructures ayant des tailles de grains très différentes. Pour la microstructure aux grains fins la solution saline accélère la fissuration et lorsque la fréquence diminue les vitesses de fissuration augmentent. Pour la microstructure grossière, la solution saline accélère aussi la propagation mais sans aucune influence de la fréquence.

\subsubsection{Influence de la fréquence et de la forme du signal}

Lors d'un cycle, la durée d'exposition de la fissure au milieu environnant dépend de la fréquence de chargement. On peut donc penser que plus la fréquence est faible, plus les interactions en pointe de fissure entre le chargement mécanique et les réactions chimiques seront importantes et pourront augmenter les vitesses de propagation. Des essais réalisés sur l'alliage d'aluminium 2024-T351 en solution saline mettent en évidence un effet opposé [89]. Entre 10 et $0.1 \mathrm{~Hz}$ les vitesses de fissuration diminuent avec la fréquence (Figure 13a,b). Cette dernière comprend en fait deux paramètres : la vitesse de chargement pendant la montée du cycle et le temps d'immersion sur un cycle. Des essais avec un chargement en dents de scie, permettant de faire varier la vitesse de chargement tout en gardant un 


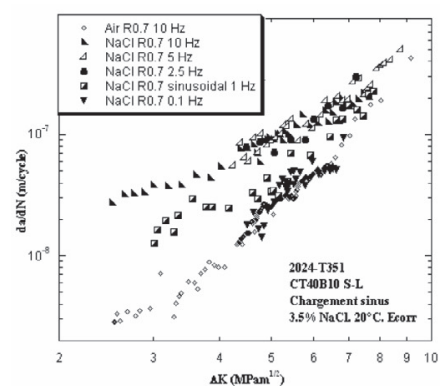

a)

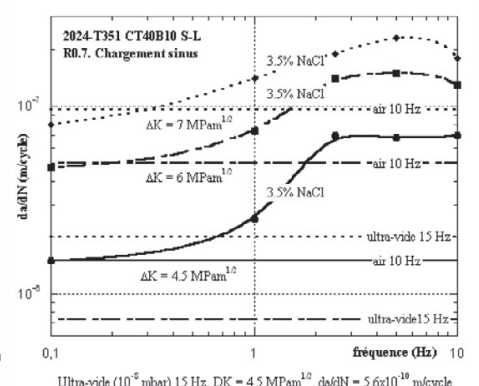

b)

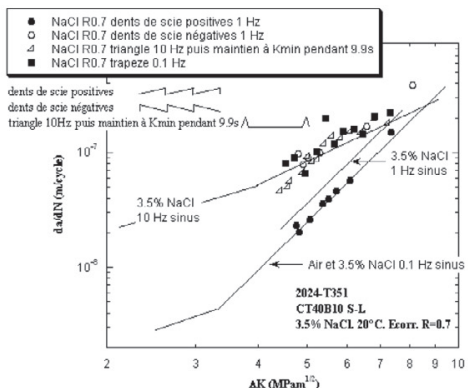

c)

Figure 13. Influence de la fréquence en solution saline pour l'alliage 2024-T351 (a) chargement sinus (b) da/dN en fonction de la fréquence à $\Delta \mathrm{K}$ constant (c) influence de la forme du signal [89].

temps d'immersion constant, montrent que c'est la vitesse de mise en charge qui contrôle en fait l'effet de fréquence observé, et qu'en revanche le temps d'immersion sur un cycle n'a que très peu d'influence sur les vitesses de fissuration (Figure 13c). On peut penser que, lorsque la vitesse de chargement est suffisamment élevée, le métal mis à nu par le glissement en pointe de fissure n'a pas le temps de se passiver et se trouve donc directement en contact avec la solution saline. Les mécanismes de fatigue corrosion (dissolution anodique, fragilisation par l'hydrogène. . . etc.) peuvent alors accélérer la fissuration si les cinétiques de réaction sont suffisamment rapides.

Cependant les tendances inverses sont plus généralement observées. Ainsi Selines et Pelloux [90] observent une forte influence de la vitesse de chargement sur les vitesses de propagation dans l'alliage 7075-T6 en solution saline, mais par contre quasiment aucune influence de la durée du cycle. Toutefois, contrairement aux résultats précédents, les vitesses de fissuration augmentent avec la vitesse de chargement. En outre, selon ces auteurs, la demie amplitude d'ouverture de la pointe de fissure $1 / 2 \times \Delta$ CTOD (Crack Tip Opening Displacement range $\triangle \mathrm{CTOD}$ ), semble constituer la limite supérieure des vitesses de propagation sous l'effet de la synergie entre le chargement cyclique et les mécanismes de corrosion. Dans cette situation extrême la loi de propagation est donnée par:

$$
\frac{\mathrm{da}}{\mathrm{dN}}=\frac{1}{2} \times \Delta \mathrm{CTOD}=\frac{1}{2} \times \frac{\Delta \mathrm{K}^{2}}{\mathrm{E} \sigma_{\mathrm{y}}}
$$

c'est-à-dire une formulation comparable au terme «hydrogène » de la formulation empirique (4). Ce résultat est souvent avancé pour décrire les changements de pente observés sur les courbes da/dN - $\Delta \mathrm{K}$ en fatigue corrosion [91, 92].

Dans le cas d'un alliage à base de fer, Wang et al. [93] ont étudié l'influence, en solution saline, des chargements sinus, dents de scie avec montée lente (positives), dents de scie avec montée rapide (négatives) et carré (long maintien à la charge maximale). Pour les quatre formes de signal, on observe une augmentation des vitesses par rapport à l'air mais l'intensité de cet effet varie considérablement d'un signal à l'autre. Ainsi dans une solution $3.5 \% \mathrm{NaCl}$ pour un $\Delta \mathrm{K}$ d'environ $25 \mathrm{MPa} \sqrt{ } \mathrm{m}$, l'écart entre les vitesses pour le chargement dents de scie positives (montée lente) est de presque un ordre de grandeur comparé aux dents de scie négatives (montée rapide).

Tous ces résultats illustrent de bien analyser l'influence de la fréquence de chargement en fatigue corrosion. En effet il convient de décomposer la fréquence en deux paramètres : la période totale du cycle, déterminant la durée d'exposition, et la vitesse de chargement. De nombreuses études font ressortir l'importance de la vitesse de chargement lorsque la synergie entre les mécanismes de fatigue et la corrosion est grande [69, 90, 93, 95], tandis que la durée d'un cycle peut avoir beaucoup d'influence lorsque la corrosion sous contrainte est susceptible de s'ajouter à l'endommagement de fatigue, en particulier si le seuil de fissuration en corrosion sous contrainte $\mathrm{K}_{\mathrm{ISCC}}$ est dépassé [78] (comportement de type b) et c) Figure 10). 


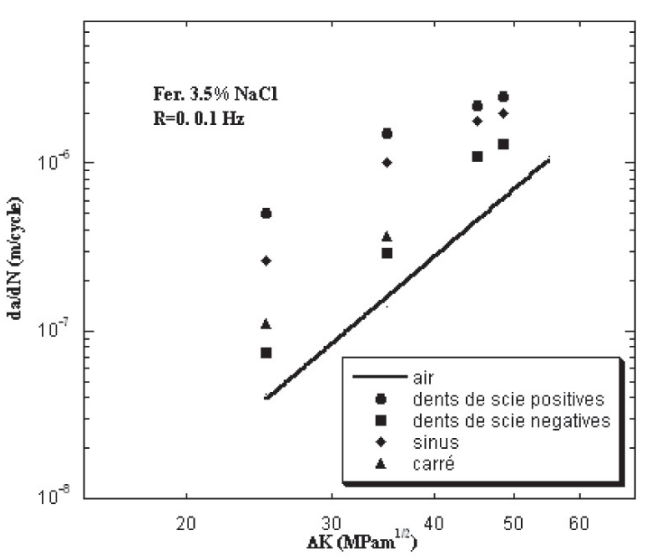

a)

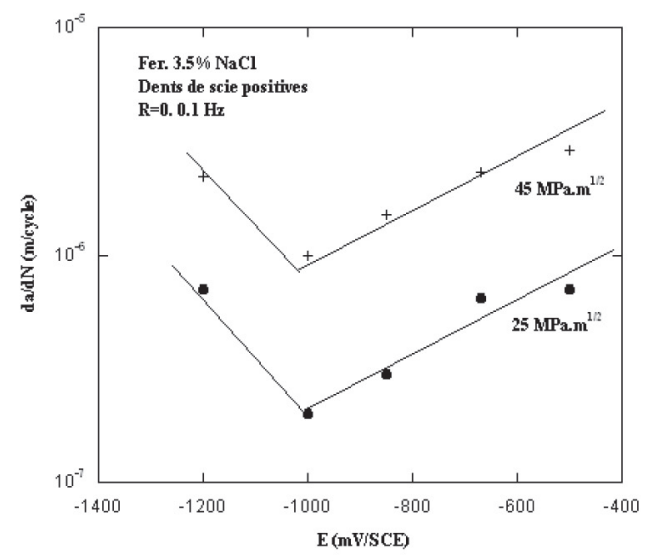

b)

Figure 14. a) Influence de la forme du signal sur un alliage à base de fer dans $3.5 \% \mathrm{NaCl}$. Potentiel électrochimique «libre»; b) influence du potentiel électrochimique (D'après Wang et al. [93, 94]).

\subsubsection{Influence des conditions électrochimiques}

Wang et al. [93] ont mesuré les vitesses de fissuration sous différentes valeurs du potentiel électrochimique $\mathrm{E}$ entre l'éprouvette et le milieu. A $\Delta \mathrm{K}$ constant, l'évolution des vitesses de fissuration en fonction du potentiel est non monotone (Figure 14b), passant par un minimum des vitesses da/dN pour un E d'environ $-1000 \mathrm{mV} / \mathrm{SCE}$. Selon les auteurs, ce type de comportement serait associé à un rôle prépondérant de la dissolution anodique pour les potentiels supérieurs à -1000 mv/SCE alors qu'en dessous de $-1000 \mathrm{mV} / \mathrm{SCE}$ ce serait les effets de fragilisation par l'hydrogène qui prédomineraient.

\section{CONCLUSIONS}

Les résultats expérimentaux présentés dans ce chapitre montrent que la propagation des fissures de fatigue dans les structures métalliques sous conditions de service peut être fortement influencée par les interactions complexes entre chargement mécanique, microstructure et milieu environnant. Ils font en outre ressortir la nécessité de bien identifier les étapes contrôlant l'ensemble du phénomène (transport en pointe, réaction de surface, diffusion) ainsi que les mécanismes d'endommagement. Toutefois cette identification est encore aujourd'hui délicate, principalement du fait de l'échelle extrêmement fine à laquelle se développent les processus de réaction et d'endommagement. Par conséquent ils ne sont pris en compte dans les modèles prédictifs que de façon largement empirique. Une modélisation plus réaliste de ces phénomènes nécessiterait, outre une meilleure compréhension et caractérisation des processus physico-chimiques en pointe de fissure, la description détaillée des couplages plasticitéendommagement prenant en compte l'action des espèces produites par les réactions de surface, notamment l'hydrogène. C'est un des verrous à lever pour garantir avec un degré de confiance accru l'extension de données d'essais accélérés à de très longues durées de vie pour différents systèmes métal/environnement.

\section{Références}

[1] Gough H.J. and Sopwith D.G., J. Inst. Met. IXL (1932) 93.

[2] Haigh B.P., J, Int. Met. 18 (1917) 55.

[3] Dahlberg E.P., Trans. A.S.M. 58 (1965) 46. 
[4] Bradshaw F.J., Scr. Metall. 1 (1967) 41.

[5] Bradshaw F.J. and Wheeler C., Appl. Mater. Res. (1966) 112.

[6] Bignonnet A., Petit J. and Zeghloul A., The influence of environment on fatigue crack growth mechanisms, (Environment Assisted Fatigue, EGF7, P. Scott Eds, Mechanical Engineering Publications, London, 1990), pp. 205.

[7] Elber W., "The Significance of Crack Closure" Damage Tolerance in Aircraft Structures, ASTM STP 486, Toronto, Ontario, Canada, 1971, (American Society for Testing and Materials, Philadelphia, Pa., 1971), pp. 230.

[8] Suresh S., Zamiski Z.A. and Ritchie R.O., Metall. Trans. A 12 (1981) 1435.

[9] Suresh S. and Ritchie R.O., "Near-threshold fatigue crack propagation: a perspective on the role of crack closure" Fatigue Crack Growth Tresholds Concepts, Philadelphia, Pennsylvania, 1983, Davidson D.L. and Sureshs S. Eds, (The Metallurgical Society of AIME, 1983), pp. 227.

[10] Petit J., De Fouquet J. and Hénaff G., Influence of ambient atmosphere on fatigue crack growth behaviour of metals, (Handbook of fatigue crack propagation in metallic structures, 2, Section VI on Influence of Environmental condition, A. Carpinteri Eds, Elsevier, 1994), pp. 1159.

[11] Liaw P.K., Hudak Jr S.J. and Donald J.K., Metall. Trans. A 13 (1982) 1633.

[12] Liaw P.K., Hudak Jr. S.J. and Donald J.K., "Near-threshold fatigue crack growth investigation of NiMoV Steel in Hydrogen Environment" Fracture mechanics: fourteenth symposium-volume II: testing and applications, ASTM STP 791, 1983, J.C. Lewis and G. Siness Eds, (American Society for Testing and Materials, 1983), pp. II.370-II.388.

[13] Weertman J., Int. J. Fract. Mech. 2 (1966) 460.

[14] McClintock, F.A. "On the Plasticity of the Growth of Fatigue Cracks" Fracture of Solids (Maple Valley WA, 1963) pp. 65.

[15] Rice J.R., "Plastic Yielding at a Crack Tip" International Conference on Fracture, Sendai, Japan, 1965) pp. 283.

[16] Tomkins B., Phil. Mag. 18 (1968) 1041.

[17] Stewart A.T., Engng. Fract. Mech. 13 (1980) 463.

[18] Suyitno B.M., Chalant G. and Petit J., "Environment and Frequency Effects on Fatigue Crack Growth of Micro-Alloyed Steel in the Threshold Region" Fatigue'90, Honolulu, Hawai, 1990, Kitagawa H. and Tanakas K. Eds, (MCEP, 1990), pp. 1381.

[19] Koterazawa R. and Nosho T., Fatigue Fract. Engng. Mater. Struct. 15 (1992) 103.

[20] Hénaff G., Petit J. and Bouchet B., Int. J. Fatigue 14 (1992) 211.

[21] Petit J., Henaff G. and Sarrazin-Baudoux C., Fatigue Crack Growth Thresholds, Endurance Limits, and Design ASTM STP 13721372 (2000) 3.

[22] Henaff G., Marchal K. and Petit J., Acta Metall. Et Mater. 43 (1995) 2931.

[23] Hénaff G., Revue de Métallurgie-CIT/Science \& Génie des Matériaux (2002) 449.

[24] Lesterlin S., Sarrazinbaudoux C. and Petit J., Scr. Mater. 34 (1996) 651.

[25] Ro Y., Agnew S.R., Bray G.H. and Gangloff R.P., Materials Science and Engineering: A The McEvily Symposium: Fatigue and Fracture of Traditional and Advanced Materials, TMS 2006 468-470 (2007) 88.

[26] Lynch S.P., Mechanisms of hydrogen assisted cracking - A review (Hydrogen Effects on Material Behavior and Corrosion Deformation Interactions, minerals, metals \& materials soc, warrendale, pa, 2003) pp. 449.

[27] Wei R.P. and Simmons G.W., Int. J. Fract. 17 (1981) 235.

[28] Achter M.R., Scr. Metall. 2 (1968) 525.

[29] Bowles C.Q., The role of environment, frequency and wave shape during fatigue crack growth in aluminium alloys. 1978, Delft University of Technology, Department of Aerospace Engineering.

[30] Donovan J.A., Met. Trans. A 7 (1976) 1677.

[31] Tien J.K., Richards R.J., Buck O. and Marcus H.L., Scrota Metall. 9 (1975) 1097.

[32] Tien J.K., Thompson A.W., Bernstein I.M. and Richards R.J., Metall. Trans. A 7 (1976) 821. 
[33] Brass A.M. and Chen J., "Hydrogen-Deformation Interactions in Iron and Nickel base Alloys" Corrosion-Deformation Interactions CDI’96, Nice, France, 1996, T. Magnins Eds (The Institue of Materials, 1996), pp. 196.

[34] Marchand N.J., Bailon J.P. and Dickson J.I., Metall. Trans. A 19 (1988) 2575.

[35] Oriani R.A., Ber. Buns. Pys. Chem. N. F. 76 (1972) 848.

[36] Oriani R.A. and Josephic P.H., Acta. metall. 22 (1974) 1065.

[37] Oriani R.A., Ann. Rev. Mater. Sci. 8 (1978) 327.

[38] Troiano A.R., Trans. ASM. 52 (1960) 54.

[39] Beachem C.D., Metall. Trans. 3 (1972) 437.

[40] Birnbaum H. K. and Sofronis P., "Mechanism for Hydrogen-Related Fracture - A Review" Corrosion-Deformation Interactions CDI'96, Nice, France, 1996 (The Institue of Materials, 1996), pp. 172.

[41] Robertson I.M. and Teter D., J. Metals. (1996) 55.

[42] Robertson I.M., Eng. Fract. Mech. 68 (2001) 671.

[43] Tabata T. and Birnbaum H.K., Scr. Metall. 18 (1984) 231.

[44] Tabata T. and Birnbaum H.K., Scr. Metall.17 (1984) 947.

[45] Birnbaum H.K. and Sofronis P., Mater. Sci. Eng. A Struct. Mater. 176 (1994) 191.

[46] Brass A.M. and Chene J., Mater. Sci. Eng. A Struct. Mater. 242 (1998) 210.

[47] Hanninen H.E., Lee T.C., Robertson I.M. and Birnbaum H.K., J. Mater. Eng. Perform. 2 (1993) 807.

[48] Lynch S.P., "A Commentary on Mechanisms of Environmentally Assisted Cracking” Corrosiondeformation Interactions CDI'96, Nice, France, 1996, T. Magnins Eds (The Institute of Metals, 1996), pp. 206.

[49] Magnin T., Isij Int. 35 (1995) 223.

[50] Magnin T., Chambreuil A. and Bayle B., Acta Mater. 44 (1996) 1457.

[51] Maier H.J. and Kæsche H., Scr. Metall. 24 (1990) 123.

[52] Magnin T., Bosch C., Wolski K. and Delafosse D., Mater. Sci. Eng. A 314 (2001) 7.

[53] McEvily A. J. and Gonzalez Velazquez J.L., Metall. Trans. A 23 (1992) 2211.

[54] Davidson D.L. and Lankford J., Int. J Fract. 17 (1981) 257.

[55] McEvily A.J., Gonzalez J.L. and Hallen J.M., Scr. Mater. 35 (1996) 761.

[56] Matsuoka S., Nishijima S., Masuda C. and Ohtsubo, S. "Near-threshold fatigue crack growth behaviors in air at room-temperature for various stainless steels" Advances in Fracture Research, New Dehli, India, 1984, S.R. Valluri, D.M.R. taplin, P. Rama Rao, J.F. Knott, and R. Dubeys, Eds (Pergamon press, 1984), pp. 1561.

[57] Li J.C M. and Liu C.T., Scr. Metall. Mater. 33 (1995) 661.

[58] Stoloff N.S. and Liu C.T., Intermetallics 2 (1994) 75.

[59] Tonneau A., Henaff G., Gerland M. and Petit J. Mater. Sci. Eng. A Struct. Mater. 256 (1998) 256.

[60] Tonneau A., Henaff G., Mabru C. and Petit J., Scr. Mater 39 (1998) 1503.

[61] Liu C.T., Lee E.H. and McKamey C.G., Scr. Metall. Mater. 23 (1989) 875.

[62] Tonneau A., Gerland M. and Henaff G., Metall. Mater. Trans. A-Phys. Metall. Mater. Sci. 32 (2001) 2345.

[63] Hénaff G. and Tonneau, A. "Environmental embrittlement of a FeAl alloy prepared by mechanical alloying under monotonic and cyclic loading” Structural Intermetallics 2001, Snow King Resort, Jackson Hole, Wy, 2001, Hemker K.J., Dimiduk D.M., Clemens H., Darolia R., Inui H., Larsen J.M., Sikka V.K., Thomas M. and Whittenbergers J.D., Eds (The Minerals, Metals \& Materials Society, 2001), pp. 571.

[64] Mabru C., Bertheau D., Pautrot S., Petit J. and Henaff G., Eng. Fract. Mech. 64 (1999) 23.

[65] Tonneau-Morel A., Henaff G. and Revue De Metallurgie-Cahiers D., Inform. Techn. 99 (2002) 401.

[66] Huneau B. and Mendez J., Int. J. Fatigue 28 (2006) 124. 
[67] Gangloff R.P., Hydrogen assisted cracking of high strength alloys, Comprehensive Structural Integrity, 6, Milne I., Ritchie R.O. and Karihaloo B., Eds, Elsevier Science, New York, 2003.

[68] Lynch S.P., "Mechanisms of Fatigue and Environmentaly Assisted Fatigue" ASTM-NBS-NSF symposium, Kansas City, 1979, J.T. Fongs eds, (ASTM STP, 1979), pp. 174-213.

[69] Ricker R.E. and Duquette D.J., "The role of environment on time dependent crack growth" Micro and macro mechanics of crack growth, Kentucky, USA, 1981, K. Sadananda, B.B. Rath, and D.J. Michels eds,1981).

[70] Ford F.P., Corrosion 52 (1996) 375.

[71] Ro Y., Agnew S.R. and Gangloff R.P., Metall. Mater. Trans. A-Phys. Metall. Mater. Sci. A 38 (2007) 3042.

[72] Ro Y., Agnew S.R. and Gangloff R.P., "Fatigue crack surface crystallography of precipitation hardened aluminum alloys" Fatigue 2006, 9th International Fatigue Congress, Atlanta, Georgia, USA, 2006, (Elsevier Ltd, 2006).

[73] Wei R.P., "On understanding environment-enhanced fatigue crack growth: a fundamental approach" ASTM-NBS symposium on Fatigue Mechanisms, Kansas City, May 1978, 1979, Fongs J.T., Eds (American Society for Testing and Materials, 1979), pp. 816.

[74] Turnbull A., Corrosion 57 (2001) 175.

[75] Sudarshan T.S., Srivatsan T.S. and Harvey D.P., Eng. Fracture Mech. 36 (1990) 827.

[76] En-Hou H. and Wei K., Corros. Sci.35 (1993) 599.

[77] Turnbull A., Corros. Sci. 23 (1983) 833.

[78] Kim S.S., Choe S.J. and Shin K.S., Metals and Materials-Korea 4 (1998) 1.

[79] Suresh S., Fatigue of Materials, Cambridge, 1991.

[80] Komai K., Murayama S. and Kanasaki H., "Corrosion-Products Induced Wedge Effect and Fatigue Crack Growth of Stainless Steels" 6th International Conference on Fracture, New Delhi, INDIA, 1984, Valluri S.R., Taplin D.M.R., Rama Rao P., Knott J.F. and Dubeys R., Eds, 1984) pp. 2489.

[81] Ruiz J., Llorca J. and Elices M., "Influence of the Environment on Fatigue Crack Growth in a SiC Reinforced Al Alloy" fatigue'93, Montreal, Quebec, Canada, 1993, Bailon J.P. and Dicksons J.I., Eds, 1993), pp. 767.

[82] Miller G.A. Hudak Jr, S.J. and Wei R.P., J. Testing Eval. 1 (1973) 524.

[83] Hudak J.S.J. and Wei R.P., Int. J. Pressure Vessels and Piping 9 (1981) 63.

[84] ASTM, Norme ASTM E647-00. Standard test method for measurement of fatigue crack growth rates. 1998.

[85] Wei R.P., Mater. Sci. Res. Int. 7 (2001) 147.

[86] Piascik R.S. and Willard S.A., Fatigue Fracture Eng. Mater. Struct. 17 (1994) 1247.

[87] Wan K., Chen G., Gao M. and Wei R., Metall. Mat. Trans. A 31 (2000) 1025.

[88] Gingell A.D.B. and King J.E., Acta Mater. 45 (1997) 3855.

[89] Menan F. and Hénaff G., LMPM-ENSMA. To be published.

[90] Selines R.J. and Pelloux R.M., Metall. Trans. 3 (1972) 2525.

[91] Tomkins B., Phil. Trans. R. Soc. Lond. Ser. A-Math. Phys. Eng. Sci. 299 (1981) 31.

[92] Speidel M.O., "stress corrosion cracking and corrosion fatigue fracture mechanics." Brown Boveri Symposia Series, 1984), pp. 85.

[93] Wang Z.F., Li J., Wang J.Q. and Ke W., Corros. Sci. 37 (1995) 1551.

[94] Jin C., Wang J.G. and Chen W.J., Scr. Metall. Mater. 32 (1995) 1579.

[95] Pelloux R.M., Fatigue-corrosion, (La fatigue des matériaux et des structures, C. Bathias and J.P. Bailon, Eds, Hermes, Paris, 1997), pp. 1365-386. 